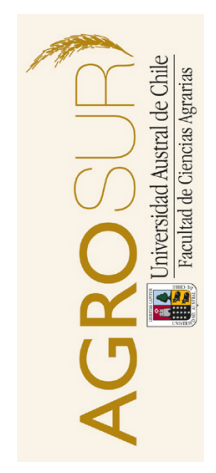

\title{
Comportamiento mecánico de un Mollisol de Chile central en función de su calidad estructural
}

\author{
Mechanical behavior of a Mollisol from central Chile \\ depending on its structural quality
}

\author{
Seguel, O. ${ }^{a *}$, Miranda, L. ${ }^{a}$, Homer, I. $^{a}$, Kremer, C. ${ }^{a}$, Kania, E. ${ }^{b}$ \\ ${ }^{a}$ Universidad de Chile, Facultad de Ciencias Agronómicas, Departamento de Ingeniería y Suelos. \\ Casilla 1004, Santiago, Chile. \\ ${ }^{b}$ Universidad de Chile, Facultad de Ciencias Agronómicas, Departamento de Producción Agrícola.
}

\begin{tabular}{l} 
A R T I C L E I N F O \\
\hline Keywords: \\
Tillage \\
Bearing capacity \\
Machinery traffic \\
Soil deformation \\
Porosity \\
\hline
\end{tabular}

Original Research Article, Soil Science

*Corresponding author:

Oscar Seguel

E-mail address:

oseguel@uchile.cl

\begin{abstract}
A B S T R A C T
In order to assess the mechanical behaviour of a soil depending on its structural quality, a Mollisol (Maipo Serie) managed with conventional tillage (LC) and meadow (P) was ploughed with a disc harrow, followed by three tractor passes over the same path. Soil samples were taken at two depths (0-20 cm and 30-50 cm) in the initial condition, after ploughing, and after the first and third tractor passes. Penetration resistance was also measured at these moments. We measured bulk density (Db), pore size distribution and consolidation test, the last one performed at two water contents (air-dry and water tension of $60 \mathrm{hPa}$ ) determining the deformation, recovery and bearing capacity. After ploughing, Db in P site decreased from 1.44 to $1.06 \mathrm{Mg} \mathrm{m}^{-3}$, and subsequent transits unchanged this value, while in the LC site, tillage caused a decrease of Db from 1.44 to $1.23 \mathrm{Mg} \mathrm{m}^{-3}$. However, subsequent transits caused consolidation, increasing the $\mathrm{Db}$ to $1.42 \mathrm{Mg} \mathrm{m}^{-3}$ in the third pass. In both soils, the coarse porosity initially increased by tillage, however, this fraction decreased with the subsequent transits in the LC site, including the 30-50 cm depth. The mechanical strength was high in both sites; the highest value was reached in LC post tillage and traffic (7 MPa). The predominance of coarse porosity in P site, determined greater sensitivity to loads and lower bearing capacity, however, the recovery was higher than in the LC site. In conclusion, the LC site showed a higher mechanical strength, but its physical properties determine a low quality for crop production.
\end{abstract}

\section{RESUMEN}

Con el objeto de evaluar el comportamiento mecánico de un suelo en función de su calidad estructural, un Mollisol (Serie Santiago) manejado bajo labranza convencional (LC) y pradera (P), fue arado y transitado por un tractor tres veces sobre la misma huella. Se tomaron muestras de suelo a dos profundidades $(0-20 \mathrm{~cm}$ y 30-50 cm) en la condición inicial, post labranza y tras el primer y tercer tránsito de tractor; también se realizaron mediciones de resistencia a la penetración. Se midió la densidad aparente (Da), la distribución de tamaño de poros y el test de consolidación en dos condiciones de humedad (seca al aire y equilibrado a -60 hPa), determinando los módulos de deformación, recuperación y capacidad de soporte. Tras la labranza, la Db disminuyó en P desde 1,41 a 1,06 $\mathrm{Mg} \mathrm{m}^{-3}$, y con los posteriores tránsitos no varió, mientras que en LC la labranza provocó una disminución de 1,44 a 1,23 $\mathrm{Mg} \mathrm{m}^{-3}$, reconsolidándose con los tránsitos hasta 1,42 $\mathrm{Mg} \mathrm{m}^{-3}$ en la tercera pasada. En ambos sitios aumentaron los poros gruesos superficiales post-labranza, sin embargo el tránsito disminuyó esta fracción en LC, incluyendo la profundidad de 30-50 cm. La resistencia mecánica fue alta para ambos sitios, con el mayor valor en LC post-labranza y con tránsito (7 MPa). El predominio de poros gruesos en P generó una mayor compresibilidad y una menor capacidad de soporte, pero la recuperación fue mayor que en el sector LC. En conclusión, LC tuvo una mayor resistencia mecánica, pero sus propiedades físicas determinan que es un suelo de menor calidad para el establecimiento vegetal.

Palabras clave: Labranza, capacidad de soporte, tránsito de maquinaria, deformación de suelo, porosidad.

\section{INTRODUCCIÓN}

La labranza consiste en la inversión y mullimiento de la capa superficial del suelo a través de araduras y rastrajes, que resultan en una disgregación que mejora las propiedades mecánicas para su posterior intervención (siembra u otro). Es así que, posterior a la labranza, la densidad aparente y la resistencia a la penetración disminuyen, aumentando la macroporosi- dad (Osunbitan et al., 2005). Sin embargo, la labranza intensiva o bajo condiciones inadecuadas de humedad (por sobre capacidad de campo) destruye la estructura del suelo, interrumpe la continuidad de los poros, reduce la cantidad de residuos presentes en la superficie y degrada la calidad del suelo (Franzluebbers, 2002). La labranza afecta la estructura del suelo por medio de la disturbación física de los agregados (Barto et al., 2010), teniendo como efecto la oxidación de la materia 
orgánica (M0), con su consecuente disminución (Six et al., 2004), afectando negativamente las propiedades físicas del suelo (Jorajuría, 2005).

Una opción para disminuir los efectos negativos de la labranza son las rotaciones con pasturas. En un estudio realizado en Uruguay, tras 12 años de rotaciones con pasturas, se obtuvo un $58 \%$ más de estabilidad de agregados al agua que sistemas de cultivos intensivos, ambos bajo labranza tradicional (Ernst y Siri-Prieto, 2009). El aumento de la estabilidad de los agregados durante la fase de pastura se debería principalmente a la ausencia de labranza, que favorece la estabilización de la MO, y al sistema fibroso y denso de las raíces de especies perennes, que genera uniones entre las partículas sólidas por amarre y por efecto cementante de los exudados (Six et al., 2004).

La labranza destruye las uniones entre partículas sólidas, disminuyendo la cohesión y la capacidad de soporte del suelo (Febo y Pessina, 2002), entendiendo a ésta como la máxima carga que puede soportar un suelo sin que presente una deformación de carácter plástico con pérdida de porosidad (Payahuala y Dörner, 2009). La zona de tránsito posterior a la labranza acerca las partículas entre sí, aumentando los puntos de contacto, lo que contribuye a recuperar la estabilidad mecánica (Horn, 2003). Esta mayor resistencia mecánica se produce a expensas de una disminución del sistema poroso, de manera que para que éste no se modifique, la presión que ejerce la maquinaria debe ser inferior a la capacidad de soporte del suelo (Schäffer et al., 2010). El efecto de la MO sobre las propiedades mecánicas del suelo apunta a aumentar su elasticidad y capacidad de soporte, previniendo la compactación, tal como lo demostraron Zhang et al. (2005), quienes comprobaron un aumento en la cohesión de las partículas, la capacidad de soporte y la recuperación elástica al agregar dosis crecientes de MO al suelo. La frecuencia de tránsito sobre un mismo punto representa otro factor importante en el comportamiento mecánico de un suelo. Posterior a la labranza, la primera pasada de un tractor tiene un efecto en profundidad mucho mayor que las siguientes, pero además la transmisión de cargas en profundidad será mayor mientras más alto es el contenido de agua (Ellies y Dörner, 1999).

Diversos estudios se han realizado respecto al efecto del uso, los sistemas de labranza y las rotaciones de cultivos sobre las propiedades físicas del suelo (Osunbitan et al., 2005; Glab y Kulig, 2008). A nivel de propiedades mecánicas, se ha estudiado la reología de los procesos de compactación asociados a las características de la maquinaria y los sistemas de labranza, desarrollando modelos de simulación y evaluación de la compactación (Arvidsson y Keller 2007); sin embargo, existen pocos antecedentes que relacionen el comportamiento mecánico del suelo y su efecto sobre las pro- piedades físicas al ser labrado y transitado, tomando como referencia un sitio poco intervenido (Dörner et al., 2013). El objetivo del presente estudio fue evaluar el comportamiento de un suelo manejado con sistema intensivo de labranza y con pradera, analizando su respuesta físico-mecánica posterior a la labranza.

\section{MATERIALES Y MÉTODO}

\section{Lugar de estudio}

El ensayo se realizó en dos sitios colindantes ubicados en la Facultad de Ciencias Agronómicas de la Universidad de Chile (3333'58" latitud S, 70³8'09" longitud 0), Comuna de La Pintana, Santiago de Chile, durante diciembre de 2014 a marzo de 2015. Uno de los sitios fue manejado con labranza intensiva (LC) y el otro correspondió a una pradera natural (P) dominada por Cynodon dactylon (L.) Pers. y Brassica sp., sin intervención en más de 5 años. Los análisis de laboratorio se realizaron en el Laboratorio de Física de Suelos de la misma Facultad.

El suelo corresponde a la Serie Maipo, miembro de la Familia franca fina, mixta, térmica, de los Fluventic Haploxerolls (CIREN, 1996). El clima es Mediterráneo, con temperatura máxima promedio en el mes más cálido (enero) de $26,2^{\circ} \mathrm{C}$, temperatura mínima en el mes más frío (julio) de $4,3^{\circ} \mathrm{C}$, precipitación promedio anual de 308,4 mm y ETo promedio anual de 992,2 mm (Uribe et al., 2012).

\section{Metodología}

\section{Caracterización inicial de los suelos}

Se realizó una caracterización de los sitios, tomando muestras compuestas entre $0-20 \mathrm{~cm}$ y $30-50 \mathrm{~cm}$, de manera de considerar el horizonte Ap (afectado por la labranza) y el horizonte subsuperficial no labrado. Se evaluó la granulometría por el método del hidrómetro de Bouyoucos y la densidad real por el método del picnómetro, ambos descritos en Sandoval et al. (2012); además se midió la materia orgánica por calcinación según método descrito en Sadzawka et al. (2006). También se evaluó la estabilidad de macroagregados y microagregados mediante los métodos de la variación del diámetro medio de los agregados (VDMA, Hartge y Horn, 2009) y la relación de dispersión (RD, Seguel et al., 2003), respectivamente.

\section{Tratamientos y diseño experimental}

En enero de 2015, con los suelos en estado friable (3 días posterior a un riego por tendido de 4 horas) se pasó en ambos sitios (LC y P) un arado de disco y una rastra tipo offset, utilizando un tractor Ford 6600. Pos- 
teriormente se hizo transitar un tractor Massey Ferguson 240 (4x2) de 46HP, 1,71 Mg y neumático trasero $14,9 \times 24$, sin implementos, tres veces por la misma huella y a una velocidad de $7 \mathrm{~km} \mathrm{~h}^{-1}$. Se consideró una presión ejercida en la zona de contacto suelo-neumático similar a la presión de inflado (Håkansson, 2005), la cual fue de $89 \mathrm{kPa}$.

En cada sitio de estudio se utilizó un diseño completamente aleatorizado (DCA) con 4 tratamientos y 4 repeticiones por tratamiento, siendo la unidad experimental una superficie de $1 \mathrm{~m}^{2}$. El tratamiento T1 correspondió a la condición o momento previo al arado de disco y rastra (llamado previo a la labranza). El tratamiento T2 correspondió al momento posterior a la labranza, denominado "0 pasadas del tractor", en tanto los tratamientos T3 y T4 correspondieron al muestreo después de la primera y tercera pasada de tractor (en la zona de tránsito de las ruedas), respectivamente.

\section{Evaluaciones}

Se midió la resistencia mecánica con un penetrómetro digital (Penetrologger, Eijkelkamp, Holanda) cada $5 \mathrm{~cm}$ hasta una profundidad de $20 \mathrm{~cm}$, y luego cada $10 \mathrm{~cm}$ hasta los $50 \mathrm{~cm}$. El resto de las propiedades se evaluaron en dos rangos de profundidad (0-20 y 30-50 $\mathrm{cm})$. Se evaluó la densidad aparente por el método del cilindro y la distribución de tamaño de poros a partir de las curvas de retención de agua, utilizando una cama de arena (Sandbox, Eijkelkamp, Holanda), ollas y platos a presión (Soil Moisture Equipment Corp, USA); en ambas pruebas se utilizaron cilindros de $5,9 \mathrm{~cm}$ de diámetro y $5 \mathrm{~cm}$ de altura (Sandoval et al., 2012). Se clasificaron los poros de acuerdo a Hartge y Horn (2009) en poros de drenaje rápido (PDR, diferencial entre equilibrios hídricos de -0,2 y -6 kPa); poros de drenaje lento (PDL, diferencial entre -6 y $-33 \mathrm{kPa}$ ); poros de agua útil (PAU, diferencial entre -33 y $-1500 \mathrm{kPa}$ ) y poros de agua inútil (PAI, retención a $-1500 \mathrm{kPa}$ ).

\section{Curva de consolidación}

Para la prueba de consolidación se duplicó el número de muestras por tratamiento, de manera de realizar las pruebas con muestras secas al aire y equilibradas a $-60 \mathrm{hPa}$; para esto se utilizaron cilindros de $10 \mathrm{~cm}$ de diámetro y $3 \mathrm{~cm}$ de altura. La prueba de consolidación se realizó con un odómetro de presión (fabricación propia) sobre muestras confinadas con drenaje libre, colocando la muestra entre dos placas metálicas porosas y aplicando una carga mediante un pistón activado por aire comprimido. La aplicación de carga consideró 9 intervalos $(20,40,60,100,150,200,300,400$ y 500 $\mathrm{kPa}$ ), dejando un tiempo de asentamiento de 3 minutos para las muestras secas al aire y 5 minutos para las muestras equilibradas a $-60 \mathrm{hPa}$, de manera de asegu- rar la máxima compresión, la cual fue medida con un comparador micrométrico. En intervalos de presión de 100, 20 y $0 \mathrm{kPa}$, se descargó la muestra para registrar la capacidad de recuperación del suelo.

Se registró la máxima deformación (mm), que corresponde al asentamiento a los $500 \mathrm{kPa}$. También se calcularon módulos de consolidación en dos tramos de la curva de consolidación: 20 a $50 \mathrm{kPa}, 300$ a $500 \mathrm{kPa}$ y el módulo de recuperación total entre los 500 y los $0 \mathrm{kPa}$ (Schäffer et al., 2010). Finalmente se calculó la capacidad de soporte con el método gráfico de Pacheco Silva, descrito por Payahuala y Dörner (2009).

\section{Análisis estadístico}

Para cada sitio y profundidad, los resultados se sometieron a un análisis de varianza (ANDEVA) con un $5 \%$ de nivel de significancia. En caso de detectar diferencias estadísticamente significativas entre los tratamientos (número de pasadas) se utilizó una prueba de comparaciones múltiples de Tukey. Para comparar diferencias de las propiedades entre sitios se utilizó una prueba $\mathrm{t}(\mathrm{P}=95 \%)$ en un mismo tratamiento y profundidad. Dado que la labranza se realizó entre $0-20 \mathrm{~cm}$ de profundidad, alterando las propiedades respecto a los 30-50 cm, este factor no se consideró dentro del análisis estadístico y sólo se utilizó como referente al comparar los sitios.

\section{RESULTADOS Y DISCUSIÓN}

\section{Caracterización inicial de los suelos}

El Cuadro 1 presenta la caracterización inicial de los sitios. Para el caso de la estabilidad de agregados, ambos métodos corresponden a pruebas de dispersión, por lo que los menores valores indican la mayor estabilidad.

Si bien el sitio LC (Labranza Convencional) presentó un menor contenido de arcilla que P (Pradera), las diferencias texturales entre sitios no son relevantes, $\mathrm{y}$ ambas se encuentran cercanas al límite de las clases $\mathrm{F}$ y FA. En cuanto a la densidad real, los valores son los esperados para suelos minerales, con niveles menores en el sitio de pradera debido al mayor contenido de MO (Sandoval et al., 2012).

Los resultados de MO son lógicos según la condición de uso y profundidad, con valores mayores en el sitio $\mathrm{P}$ entre los $0-20 \mathrm{~cm}$ debido al desarrollo de la pradera. $\mathrm{Al}$ incluir pasturas en la rotación de cultivos en un Typic Argiudoll, Salvo et al. (2010) registraron un aumento de un $23 \%$ en el nivel de carbono orgánico del suelo, comparado con un sistema intensivo, ambos bajo labranza tradicional. Por otro lado, al calcular el índice de estratificación de la MO (Franzluebbers, 2002) el cual corresponde al cociente entre los contenidos de $\mathrm{MO}$ su- 
Cuadro 1. Textura, densidad real (Dr), materia orgánica (MO) y estabilidad de agregados para ambos tratamientos (LC: Labranza convencional; P: Pradera). Valores iniciales de RD obtenidos con muestra compuesta.

Table 1. Texture, particle density (Dr), organic matter (MO) and aggregate stability for both treatments (LC: conventional tillage; P: grassland). Initial values of RD obtained with composite sample.

\begin{tabular}{ccccccccc}
\hline Sitio & $\begin{array}{c}\text { Prof. } \\
\mathbf{( c m )}\end{array}$ & $\begin{array}{c}\mathbf{A} \\
\mathbf{( \% )}\end{array}$ & $\begin{array}{c}\mathbf{L} \\
\mathbf{( \% )}\end{array}$ & $\begin{array}{c}\mathbf{a} \\
\mathbf{( \% )}\end{array}$ & $\begin{array}{c}\mathbf{D r} \\
\mathbf{( M g ~ m}^{-3} \mathbf{)}\end{array}$ & $\begin{array}{c}\text { Mo } \\
\mathbf{( \% )}\end{array}$ & $\begin{array}{c}\text { VDMA } \\
(\mathbf{m m})\end{array}$ & $\begin{array}{c}\text { RD } \\
\mathbf{( \% )}\end{array}$ \\
\hline \multirow{2}{*}{ LC } & $0-20$ & 26,0 & 37,6 & 36,4 & 2,74 & $2,89( \pm 0,36)$ & $5,34( \pm 1,55)$ & 65,5 \\
& $30-50$ & 22,3 & 33,6 & 44,1 & 2,74 & $2,15( \pm 0,04)$ & $3,95( \pm 0,47)$ & 44,8 \\
& $0-20$ & 32,3 & 36,2 & 31,5 & 2,64 & $4,32( \pm 0,06)$ & $0,42( \pm 0,26)$ & 51,1 \\
P & $30-50$ & 34,5 & 34,1 & 31,4 & 2,66 & $2,84( \pm 0,05)$ & $3,15( \pm 0,27)$ & 69,8 \\
\hline
\end{tabular}

A: arcilla; L: limo; a: arena; VDMA: Variación de diámetro medio de agregados; RD: Relación de dispersión.

perficial y en profundidad, se obtienen valores de 1,35 y 1,52 para los sitios LC y P respectivamente, situando al suelo del sitio P con una mayor calidad. En los casos presentados por Franzluebbers (2002), los suelos bajo labranza convencional presentaron índices menores a 2 , por lo que los valores obtenidos son esperables si se considera que el sitio de pradera alguna vez fue usado agrícolamente.

Con respecto a la estabilidad de los macroagregados, en el sitio LC se ha producido una pérdida de MO respecto al sitio $\mathrm{P}$, lo que se tradujo en una alta VDMA (baja estabilidad) en el horizonte superficial del sitio LC. Si bien se espera que exista una relación inversa entre MO y VDMA en el caso del sitio LC, es posible que el corto tiempo de permanencia de la MO en la capa superficial no permita que ésta cumpla su función de agente agregante (Chenu y Plante, 2006). Por su parte, la MO medida en profundidad correspondería a un material más humificado, lo que en el sitio LC genera un menor valor de VDMA (mayor estabilidad).

En cuanto a la estabilidad de microagregados, el sitio $\mathrm{P}$ posee una menor RD (menor dispersión) en superficie que en profundidad y que en el respectivo sitio LC. Esto es resultado del mayor contenido de MO, aportado principalmente por el sistema fibroso de raíces que se produce bajo esta condición (Ernst y Siri-Prieto, 2009). Sin embargo, en profundidad el comportamiento es totalmente inverso entre sitios, aunque sigue la misma tendencia que su respectivo VDMA. Esto indicaría que para ambos tipos de agregados (macro y micro), en el sitio LC la labranza generó la protección de la $\mathrm{MO}$ en profundidad, pero en el caso de $\mathrm{P}$, el efecto dependería de la actividad del sistema de raíces, generándose la mayor estabilidad en superficie, tal como lo demostró Seguel et al. (2011) al utilizar una rotación de cultivos de cobertura en un parronal de uva de mesa, donde el riego genera un crecimiento superficial de las raíces del cultivo, sin una suficiente exploración en profundidad.

\section{Efecto de la labranza y el tránsito de maquinaria}

\section{Densidad aparente}

El Cuadro 2 presenta los valores de densidad aparente (Da), donde se observó que, inmediatamente después de realizada la labranza, se obtiene el resultado esperado, con la soltura mecánica de partículas y agregados.

Cuadro 2. Densidad aparente para cada tratamiento, sitio y profundidad. Valores promedio $( \pm \mathrm{DE})$.

Table 2. Bulk density for each treatment, site and depth. Average values $( \pm$ SD).

\begin{tabular}{ccc}
\hline $\mathrm{N}^{\circ}$ Pasadas & \multicolumn{2}{c}{ Densidad aparente $\left(\mathrm{Mg} \mathrm{m}^{-3}\right)$} \\
\cline { 2 - 3 } & \multicolumn{2}{c}{ LC } \\
\hline \multirow{2}{*}{ Previa } & \multicolumn{2}{c}{ Superficie $(0-20 \mathrm{~cm})$} \\
\cline { 2 - 3 } 0 & $1,44( \pm 0,16) \mathrm{a}$ & $1,41( \pm 0,05) \mathrm{a}$ \\
1 & $1,23( \pm 0,07) \mathrm{b}$ & $1,06( \pm 0,05) \mathrm{b}^{*}$ \\
3 & $1,42( \pm 0,05) \mathrm{ab}$ & $1,06( \pm 0,03) \mathrm{b}^{*}$ \\
\hline & \multicolumn{2}{c}{ Profundidad $(30-50 \mathrm{~cm})$} \\
\cline { 2 - 3 } Previa & $1,45( \pm 0,13) \mathrm{a}$ & $1,37( \pm 0,02) \mathrm{a}$ \\
0 & $1,47( \pm 0,06) \mathrm{a}$ & $1,44( \pm 0,08) \mathrm{a}$ \\
1 & $1,43( \pm 0,06) \mathrm{a}$ & $1,41( \pm 0,04) \mathrm{a}$ \\
3 & $1,52( \pm 0,11) \mathrm{a}$ & $1,42( \pm 0,08) \mathrm{a}$ \\
\hline
\end{tabular}

Promedios unidos por letras minúsculas distintas en sentido vertical indican diferencias estadísticamente significativas entre tratamientos $\left(\mathrm{n}^{\circ}\right.$ de pasadas) dentro de cada sitio y profundidad, según el test de Tukey (p-valor $<0,05)$. Asterisco $\left({ }^{*}\right)$ indica diferencias estadísticamente significativas en sentido horizontal entre los sitios en cada $n^{\circ}$ de pasadas y profundidad. LC: Labranza convencional; P: Pradera. 
En LC se observó una evolución de la Da acorde a los tratamientos, ya que la labranza disturba el suelo, separa los agregados y aumenta el porcentaje de poros; el tránsito posterior de la maquinaria acerca las partículas y las comprime, disminuyendo parte del espacio poroso generado previamente (Alleto y Coquet, 2009). La tercera pasada tiende a acercar aún más las partículas, llegando a un punto de estabilidad para la carga aplicada, tal como lo demostraron Usowicz y Lipiec (2009) en un suelo franco limoso, donde la Da mostró la misma dinámica en respuesta al tránsito de maquinaria. En el caso de P, se aprecia un mayor efecto disturbador de la labranza, que alcanzó menores valores de Da en relación a LC, existiendo diferencias significativas entre los sitios incluso hasta la tercera pasada de tractor. Lo anterior se debe a la mejor calidad estructural del sitio $P$, ya que de acuerdo a Franzluebbers (2002), la estratificación de la MO permite conservar la integridad de los agregados incluso posterior a la labranza, lo que potencia la formación de espacios interagregados estables.

En el caso de la Da en profundidad, si bien no hubo diferencias significativas entre tratamientos, existe una tendencia al aumento inmediatamente después de la labranza, lo que se puede deber a la transmisión en profundidad de la presión ejercida por el implemento de labranza (Jorajuría, 2005). Sin embargo, al evaluar el efecto de 16 pasadas de tractor, Ellies y Dörner (1999) determinaron que con cada pasada el suelo se compacta superficialmente, disminuyendo la profundidad de penetración de las tensiones, por lo que el efecto sobre la Da en profundidad es marginal.

\section{Distribución de tamaño de poros}

Los resultados de la distribución de tamaño de poros se presentan en el Cuadro 3.

La labranza interviene el suelo, disgregándolo en agregados, lo que genera un aumento del espacio entre éstos. En el Cuadro 3 es posible ver este efecto, donde en superficie y tras la labranza (0 pasadas de tractor), ambos sitios aumentaron el porcentaje de macroporos (poros de drenaje rápido, $\mathrm{PDR},>50 \mu \mathrm{m}$ y poros de dre-

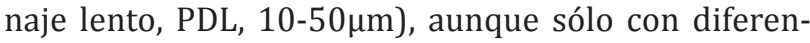
cias estadísticamente significativas en el sitio LC para los PDL. Horn y Fleige (2009) plantean un mínimo de $8 \%$ de PDR para asegurar adecuado intercambio gaseoso en el suelo. En este sentido, el sitio P, tras ser transitado, es capaz de mantener una proporción adecuada de PDR, no así el sitio LC, que con el tránsito se asienta de manera considerable. Por otro lado, el bajo porcentaje inicial de PDR en el suelo del sitio P, se debe a que estos poros son rellenados parcialmente con MO, lo que sucede comúnmente en suelos con aportes continuos de MO y alta actividad biológica (Seguel y Horn, 2006a).
Al transitar una vez sobre el suelo recién preparado, es posible observar que en LC se conservan los PDL pero disminuyen de manera significativa los PDR, mientras que P conserva los PDR y PDL ganados por la labranza. Este comportamiento se debe a la estabilidad de los agregados, la cual es mayor en P (Cuadro 2) como consecuencia de la protección física de la MO (Mulumba y Lal, 2008). Así, un manejo que genera la acumulación de MO, promueve un aumento de la friabilidad del volumen suelo, con agregados más densos y estables desde el punto de vista físico y mecánico, lo que le permite mantener un sistema macroporoso por más tiempo (Chenu y Plante, 2006).

La transmisión de la presión en profundidad (30$50 \mathrm{~cm}$ ) afecta de manera diferente a los macroporos en función del sitio. Es así como en LC se produce una disminución significativa de PDR, mientras que en P disminuyen de manera significativa los PDL. El roce de los implementos al momento de realizar la labranza es el factor que explica el proceso de compresión de estos poros (Jorajuría, 2005), ya que los posteriores tránsitos no tienen mayor efecto. La condición previa a la labranza responde a la condición final de la temporada anterior, con $n$ ciclos de secado y humedecimiento que han contribuido a generar estructura (Seguel y Horn, 2006b), por lo que el paso de la maquinaria con el implemento de labranza genera una reacción en profundidad que difiere según el uso previo, destacando la baja estabilidad del sitio LC, que disminuye su porosidad gruesa (PDR, $>50 \mu \mathrm{m}$ ) por debajo del $8 \%$, señalado por Horn y Fleige (2009) como adecuado para el intercambio gaseoso.

En cuanto a los poros de agua útil (PAU, 0,2-10 $\mu \mathrm{m}$ ), a nivel superficial $(0-20 \mathrm{~cm})$ no se encontraron diferencias significativas entre tratamientos, pero sí una tendencia a la disminución en LC y al aumento en P posterior a la labranza, lo que podría estar relacionado principalmente al reacomodo de microagregados en LC como consecuencia de su baja estabilidad física mecánica (Seguel y Horn, 2006b) y a la elasticidad frente a cargas normales en P (Schäffer et al., 2007). Por otro lado, los poros de agua inútil (PAI, $<0,2 \mu \mathrm{m}$ ) disminuyeron después de la labranza por efecto de la soltura de la tensión normal propia del peso del suelo (Horn et al., 2007) y aumentaron acorde a las pasadas de tractor, llegando finalmente a valores cercanos a la condición inicial, con diferencias significativas entre los sectores, donde P presentó una mayor microporosidad acorde al mayor contenido de arcilla (Warrick, 2002).

En profundidad (30-50 cm), el colapso de los PDR en LC permite el aumento significativo de los PAU, llegando ambos casos (LC y P) a niveles adecuados según Reynolds et al. (2009), en tanto el mayor contenido de arcilla favorece la densificación en profundidad en el sitio P (Håkansson, 2005), con un incremento de los PAI. 


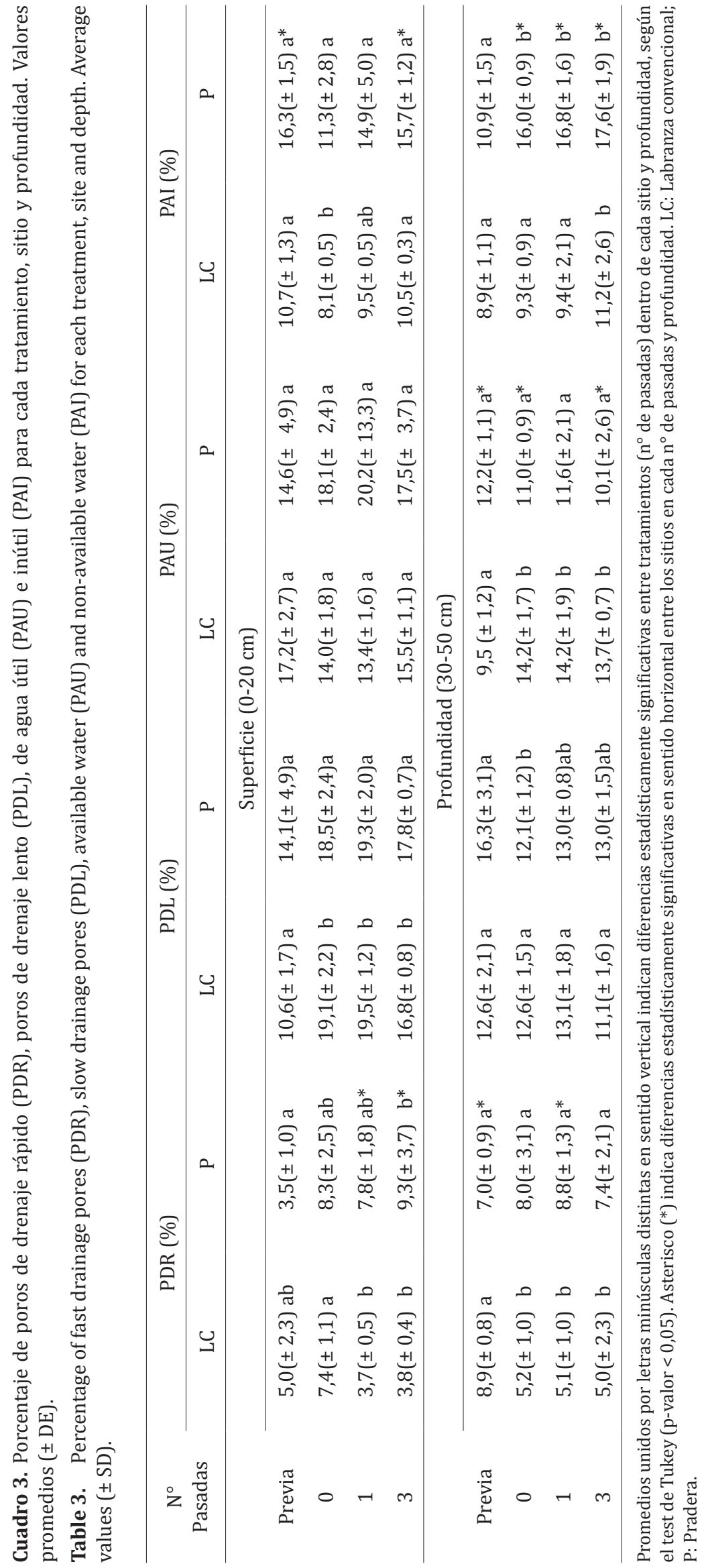




\section{Propiedades mecánicas del suelo}

\section{Resistencia a la penetración}

La resistencia a la penetración en ambos sitios presenta un comportamiento similar en su condición inicial (Cuadro 4), sin embargo se observan diferencias aproximadamente por debajo de los $15 \mathrm{~cm}$ de profundidad, donde la resistencia en el sitio P aumenta gradualmente, mientras que en el sitio LC se denota la existencia de un pie de arado entre $15-30 \mathrm{~cm}$.

Tras la labranza, la resistencia en el sitio $P$ no se ve afectada, mientras que en el sitio LC tiene una disminución hasta los $20 \mathrm{~cm}$, aproximadamente (zona de labranza), aunque sin diferencias estadísticas significativas con respecto a la condición inicial. En profundidad se observa un aumento fuerte de la resistencia, aunque esto último correspondería a una distribución irregular del contenido de agua post-labranza, lo que genera cambios en la resistencia mecánica (Vaz et al., 2011). Las raíces abundantes en $\mathrm{P}$ disminuyeron el efecto dis- turbador en profundidad de la aradura, permitiendo conservar macrounidades de suelo que mantuvieron la resistencia inicial; así, con el primer tránsito en el sitio P se disminuye la resistencia por destrucción de estas macrounidades (Six et al., 2004). Tras la tercera pasada de tractor, en el sitio $\mathrm{P}$ aumenta la resistencia en los primeros $15 \mathrm{~cm}$, alcanzando valores parecidos a la condición inicial; sin embargo en profundidad la resistencia es notablemente menor comparado con la condición inicial. Por otro lado, en el sitio LC también aumenta la resistencia en los primeros $15 \mathrm{~cm}$, con valores similares a la condición inicial, pero la resistencia en profundidad aumenta de manera considerable en comparación con la condición pre-labranza.

Al aplicar una carga mecánica sobre el suelo, se produce una alteración del volumen de éste, principalmente producto de la pérdida de poros ocupados por aire, aumentando la proporción de agua, lo que modifica la estabilidad de la matriz suelo (Mordhorst et al., 2012). Dependiendo de la magnitud de la carga y de la continuidad del sistema poroso, el agua puede actuar entregan-

Cuadro 4. Resistencia a la penetración para el sitio con pradera (p) y labranza convencional (LC). Valores promedio ( \pm DE).

Table 4. Penetration resistance in meadowsite (P) and conventional tillage (LC). Average values $( \pm$ SD).

\begin{tabular}{|c|c|c|c|c|c|c|c|}
\hline \multirow{3}{*}{$\begin{array}{c}\mathrm{N}^{\circ} \\
\text { Pasadas }\end{array}$} & \multicolumn{7}{|c|}{ Profundidad $(\mathrm{cm})$} \\
\hline & $0-5$ & $5-10$ & $10-15$ & $15-20$ & $20-30$ & $30-40$ & $40-50$ \\
\hline & \multicolumn{7}{|c|}{ Resistencia (kPa) } \\
\hline $\begin{array}{c}\mathrm{P} \\
\text { inicial }\end{array}$ & $\begin{array}{l}1042 \mathrm{a} \\
\pm \pm 196)\end{array}$ & $\begin{array}{l}2488 a^{*} \\
( \pm 433)\end{array}$ & $\begin{array}{l}3274 \mathrm{a} \\
( \pm 677)\end{array}$ & $\begin{array}{l}3935 \mathrm{ab} \\
( \pm 944)\end{array}$ & $\begin{array}{c}4461 \mathrm{a} \\
( \pm 1276)\end{array}$ & $\begin{array}{l}5356 \mathrm{a}^{*} \\
( \pm 709)\end{array}$ & $\begin{array}{c}6193 \mathrm{a} \\
( \pm 1310)\end{array}$ \\
\hline P 0 & $\begin{array}{l}640 a^{*} \\
\pm \pm 168)\end{array}$ & $\begin{array}{l}1485 \mathrm{ab} \\
( \pm 849)\end{array}$ & $\begin{array}{l}3837 a^{*} \\
( \pm 1549)\end{array}$ & $\begin{array}{l}4704 \mathrm{a}^{*} \\
( \pm 1440)\end{array}$ & $\begin{array}{l}4558 \mathrm{a} \\
( \pm 772)\end{array}$ & $\begin{array}{c}6248 \mathrm{a} \\
( \pm 1360)\end{array}$ & $\begin{array}{l}5770 a^{*} \\
( \pm 280)\end{array}$ \\
\hline P 1 & $\begin{array}{c}619 \mathrm{a} \\
( \pm 638)\end{array}$ & $\begin{array}{l}1154 \mathrm{~b} \\
( \pm 571)\end{array}$ & $\begin{array}{l}1761 \mathrm{~b} \\
( \pm 378)\end{array}$ & $\begin{array}{l}2476 \mathrm{bc} \\
( \pm 533)\end{array}$ & $\begin{array}{c}3914 \mathrm{ab}^{*} \\
( \pm 762)\end{array}$ & $\begin{array}{l}5943 \mathrm{a}^{*} \\
( \pm 801)\end{array}$ & $\begin{array}{c}6021 \mathrm{a} \\
( \pm 1190)\end{array}$ \\
\hline P3 & $\begin{array}{c}644 \mathrm{a} \\
( \pm 115)\end{array}$ & $\begin{array}{l}2287 \mathrm{ab} \\
( \pm 695)\end{array}$ & $\begin{array}{l}2477 \mathrm{ab} \\
\pm(338)\end{array}$ & $\begin{array}{l}2220 \mathrm{c} \\
( \pm 400)\end{array}$ & $\begin{array}{l}2705 b^{*} \\
( \pm 535)\end{array}$ & $\begin{array}{c}4537 a \\
( \pm 1870)\end{array}$ & $\begin{array}{l}4468 \mathrm{a} \\
( \pm 432)\end{array}$ \\
\hline $\begin{array}{c}\text { LC } \\
\text { inicial }\end{array}$ & $\begin{array}{c}638 \mathrm{a} \\
( \pm 443)\end{array}$ & $\begin{array}{l}1479 \mathrm{a} \\
( \pm 770)\end{array}$ & $\begin{array}{l}2675 \mathrm{a} \\
( \pm 1673)\end{array}$ & $\begin{array}{c}4038 \mathrm{a} \\
( \pm 1980)\end{array}$ & $\begin{array}{l}3807 \mathrm{a} \\
\pm 816)\end{array}$ & $\begin{array}{c}2030 \\
( \pm 622)\end{array}$ & $\begin{array}{c}3058 \\
( \pm 2577)\end{array}$ \\
\hline LC 0 & $\begin{array}{c}414 \mathrm{a} \\
( \pm 139)\end{array}$ & $\begin{array}{l}1047 a \\
( \pm 392)\end{array}$ & $\begin{array}{l}1636 \mathrm{a} \\
( \pm 893)\end{array}$ & $\begin{array}{c}1565 \mathrm{a} \\
( \pm 1116)\end{array}$ & $\begin{array}{c}4982 \mathrm{a} \\
( \pm 2114)\end{array}$ & $\begin{array}{c}8026 \\
( \pm 1858)\end{array}$ & 8220 \\
\hline LC 1 & $\begin{array}{l}470 \mathrm{a} \\
( \pm 104)\end{array}$ & $\begin{array}{c}851 \mathrm{a} \\
( \pm 254)\end{array}$ & $\begin{array}{l}1523 \text { a } \\
( \pm 719)\end{array}$ & $\begin{array}{l}2376 \mathrm{a} \\
( \pm 898)\end{array}$ & $\begin{array}{c}6848 \mathrm{a} \\
( \pm 1101)\end{array}$ & $\begin{array}{c}9196 \\
( \pm 1101)\end{array}$ & --- \\
\hline LC 3 & $\begin{array}{c}472 \mathrm{a} \\
( \pm 151)\end{array}$ & $\begin{array}{l}1307 \mathrm{a} \\
( \pm 824)\end{array}$ & $\begin{array}{c}2751 \mathrm{a} \\
( \pm 1616)\end{array}$ & $\begin{array}{c}4165 \mathrm{a} \\
( \pm 2697)\end{array}$ & $\begin{array}{c}6547 \mathrm{a} \\
( \pm 2717)\end{array}$ & $\begin{array}{c}5990 \\
( \pm 3366)\end{array}$ & 3173 \\
\hline
\end{tabular}

Letras distintas en sentido vertical indican diferencias estadísticamente significativas entre tratamientos $\left(\mathrm{n}^{\circ}\right.$ de pasadas) dentro de cada manejo y en cada rango profundidad, según el test de Tukey ( $\mathrm{p}$-valor $<0,05$ ).

Asterisco $\left(^{*}\right)$ indica diferencias entre manejos para el mismo tratamiento y en cada rango de profundidad $(\mathrm{p}$-valor $<0,05)$.

--- No se logró medir 
do estabilidad, en caso que se mantenga bajo tensión, o disminuyendo la estabilidad, al actuar como un agente lubricante, lo que modifica la resistencia mecánica (Seguel y Horn, 2006b). Esto es lo que posiblemente ocurre tras la tercera pasada en el sitio P entre 15 y $30 \mathrm{~cm}$, donde la transmisión de la cargas altera la proporción de agua, lo que produce el colapso de los agregados y por ende la disminución de la resistencia (Nearing, 1995).

Taylor y Brar (1991) señalan que el valor crítico de resistencia sobre el cual se reduce la elongación radical es de $2 \mathrm{MPa}$, en tanto Schoeneberger et al. (2012) señalan valores mayores a $4 \mathrm{MPa}$ como muy altos y mayores a $8 \mathrm{MPa}$ como extremadamente altos. Es decir, ambos suelos en general ofrecen resistencias altas para el crecimiento de las raíces, destacando el sitio LC post labranza y con un tránsito, donde se alcanzaron valores superiores a los $7 \mathrm{MPa}$.

\section{Curva de consolidación}

Las curvas de consolidación desarrollan una pérdida gradual de porosidad (aumento del asentamiento) a medida que aumenta la carga normal (Figura 1), mostrando diferencias según contenido de agua y condición estructural.

En la condición previa a la labranza, el suelo posee una estructura estable, por lo que resiste las cargas externas sin deformarse tanto en comparación con la condición post-labranza (Schäffer et al., 2020). En ambas condiciones de humedad se observa que la consolidación tiene una mayor pendiente (deformación) post labranza, ya que al dejar el suelo suelto y poroso, las presiones se transmiten concentradas bajo el centro de carga, permitiendo un mayor reacomodo de partículas (Usowicz y Lipiec, 2009).

Por otra parte, al comparar el comportamiento de las muestras equilibradas a $-60 \mathrm{hPa}$ y secas al aire, se puede ver el efecto del agua en la estabilidad mecánica, debido a la generación de tensiones internas, producto del secado del suelo (Schäffer et al., 2007). Es así que al humectarse las partículas, el agua actúa como lubricante, favoreciendo la mayor deformación (Tang et al., 2009). Además, la prueba de consolidación se realizó con la muestra confinada con drenaje libre, por lo que se permite disipar la presión de las cargas aplicadas mediante el flujo de agua hacia el exterior, lo que resulta en una deformación más violenta y en un asentamiento mayor que las muestras secas al aire.

\section{Máxima deformación}

Al analizar la máxima deformación superficial del sitio LC (Cuadro 5), se ve el efecto que tiene el agua sobre un suelo pobre en estructuración, ya que al equilibrar las muestras a $-60 \mathrm{hPa}$, el proceso de hidratación provoca el colapso del sistema poroso hasta una condición de homogenización (Chenu y Plante, 2006). Esta condición se repitió en estado seco al aire para el sitio LC, donde la poca estructuración genera una baja estabilidad mecánica, con un asentamiento inmediatamente después de la labranza. En condición seca al aire, pese a no mostrar diferencias significativas, el tratamiento de 0 pasadas del sitio LC es el que alcanzó la mayor deformación, lo que era esperable debido

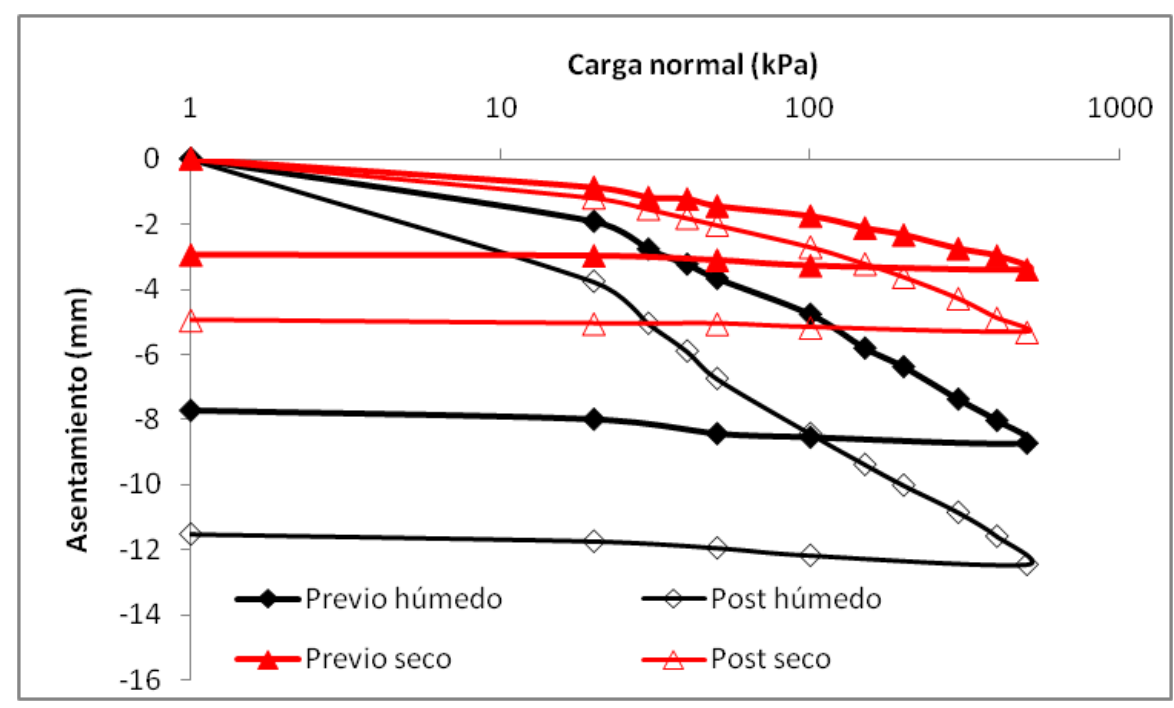

Figura 1. Curvas de consolidación típicas del sitio de pradera previo y posterior a la labranza, en dos condiciones de humedad $(-60 \mathrm{hPa}$ y seco al aire).

Figure 1. Tipical consolidation curves for grassland site before and after tillage, in two moisture conditions (-60 hPa and airdried). 
Cuadro 5. Máxima deformación $\left(\sigma_{n}=500 \mathrm{kPa}\right)$ en muestras equilibradas a -60 hPa y secas al aire, para cada tratamiento, sitio y profundidad. Valores promedios $( \pm \mathrm{DE})$.

Table 5. Maximum deformation $\left(\sigma_{\mathrm{n}}=500 \mathrm{kPa}\right)$ in samples equilibrated at $-60 \mathrm{hPa}$ and air-dried, for each treatment, site and depth. Average values $( \pm$ SD).

\begin{tabular}{|c|c|c|c|c|}
\hline \multirow{2}{*}{$\begin{array}{c}\mathrm{N}^{\circ} \\
\text { Pasadas }\end{array}$} & \multicolumn{2}{|c|}{$-60 \mathrm{hPa}(\mathrm{mm})$} & \multicolumn{2}{|c|}{ Seco al aire $(\mathrm{mm})$} \\
\hline & $\mathrm{LC}$ & $\mathrm{P}$ & LC & $\mathrm{P}$ \\
\hline & \multicolumn{4}{|c|}{ Superficie $(0-20 \mathrm{~cm})$} \\
\hline Previa & $-7,75( \pm 1,58)$ a & $-8,72( \pm 1,55)$ a & $-4,82( \pm 1,30)$ a & $-3,38( \pm 0,45)$ a \\
\hline 0 & $-6,75( \pm 2,34)$ a & $-12,44( \pm 1,17) b^{*}$ & $-5,49( \pm 0,66)$ a & $-5,31( \pm 0,50) b$ \\
\hline 1 & $-7,99( \pm 1,26)$ a & $-12,25( \pm 0,90) b^{*}$ & $-4,31( \pm 1,01) \mathrm{a}$ & $-6,22( \pm 0,73) b^{*}$ \\
\hline \multirow[t]{2}{*}{3} & $-7,62( \pm 0,62)$ a & $-12,30( \pm 0,67) b^{*}$ & $-4,76( \pm 1,15) \mathrm{a}$ & $-5,17( \pm 0,57) b$ \\
\hline & \multicolumn{4}{|c|}{ Profundidad $(30-50 \mathrm{~cm})$} \\
\hline Previa & $-7,73( \pm 1,92) \mathrm{a}$ & $-7,19( \pm 1,10) \mathrm{a}$ & $-3,67( \pm 1,62)$ a & $-3,93( \pm 1,09)$ a \\
\hline 0 & $-7,10( \pm 0,48) a$ & $-9,94( \pm 1,44) \quad b^{*}$ & $-3,39( \pm 1,15) \mathrm{a}$ & $-4,26( \pm 1,16) \mathrm{a}$ \\
\hline 1 & $-6,90( \pm 1,01)$ a & $-10,47( \pm 0,96) \quad b^{*}$ & $-2,17( \pm 0,43)$ a & $-5,25( \pm 1,00) a^{*}$ \\
\hline 3 & $-5,84( \pm 1,32) \mathrm{a}$ & $-9,00( \pm 0,93) a b^{*}$ & $-3,22( \pm 0,54) \mathrm{a}$ & $-5,02( \pm 0,89) a^{*}$ \\
\hline
\end{tabular}

Promedios unidos por letras minúsculas distintas en sentido vertical indican diferencias estadísticamente significativas entre tratamientos ( $\mathrm{n}^{\circ}$ de pasadas) dentro de cada sitio y profundidad, según el test de Tukey ( $\mathrm{p}$-valor $\left.<0,05\right)$. Asterisco $(*)$ indica diferencias estadísticamente significativas en sentido horizontal entre los sitios en cada $\mathrm{n}^{\circ}$ de pasadas y profundidad. LC: Labranza convencional; P: Pradera.

al espacio inter-agregados que genera la labranza. Al transitar sobre el suelo preparado, se pierde el espacio poroso, y como resultado las cargas aplicadas en la prueba de laboratorio producen un menor asentamiento (Fuentes et al., 2013a).

En el caso del sitio P, existen diferencias estadísticamente significativas entre la condición pre y post labranza en ambas condiciones de equilibrio mátrico y en la muestra húmeda de 30-50 cm. Esto puede estar dado por el aumento considerable de macroporosidad que genera la labranza en el sitio P (Cuadro 3); así, al ser sometida a cargas constantes y crecientes (prueba de laboratorio), la macroporosidad colapsa, logrando un asentamiento considerablemente mayor que el sitio LC. En profundidad se manifiestan mucho menos diferencias por efecto del paso de la maquinaria, ya que al no existir una soltura mecánica, la capacidad de deformación responde a un manejo con $n$ cargas del tránsito de la temporada previa, incluso en sectores con baja intensidad de tránsito (Rücknagel et al., 2007).

Las diferencias significativas que se encuentran entre sitios se relacionan con el porcentaje de macroporos presentes en cada caso (Cuadro 3), lo que se repite en las muestras que fueron extraídas en profundidad. Al correlacionar ambas variables se genera un ajuste lineal con una significancia cercana al 99\% para el caso de las muestras húmedas (Def $=-0,63 \cdot$ macroporos 4,65 ) y del $95 \%$ para el caso de las muestras secas al aire $($ Def $=-0,255 \cdot$ macroporos $-2,74)$.

\section{Módulo de deformación entre 20 y $50 \mathrm{kPa}$}

En el sitio LC equilibrado a $-60 \mathrm{hPa}$ no existe gran variación en los módulos de deformación (Cuadro 6), con valores similares entre tratamientos en ambas profundidades. En condición seca al aire, si bien los módulos disminuyen, tampoco presentaron diferencias entre tratamientos. En el sitio $\mathrm{P}$ en la condición húmeda, se produjo un aumento significativo de este módulo posterior a la labranza $(0-20 \mathrm{~cm})$, con diferencias significativas respecto al sitio LC. En profundidad, si bien no se generan diferencias significativas entre tratamientos, sí existe la misma tendencia.

En las muestras superficiales secas al aire de P, la tercera pasada de tractor igualó el módulo de deformación de LC, incluso con una primera pasada que generó diferencias significativas entre los sitios. El sitio $\mathrm{P}$, al tener una mejor calidad de agregados, tiene la capacidad de disminuir el efecto de la consolidación entre la primera y la tercera pasada, a diferencia del sitio LC, en el cual el tránsito constante aumenta su módulo de deformación. Las cargas sucesivas en LC continúan destruyendo agregados (por su baja estabilidad físicomecánica), en cambio $\mathrm{P}$ pierde los macroporos entre los agregados, pero mantiene agregados intactos que manifiestan elasticidad por su mayor contenido de MO (Zhang et al., 2005). Lo anterior se repite en profundidad, sin embargo, el sitio LC alcanza módulos significativamente más bajos que el sitio $\mathrm{P}$. 
Cuadro 6. Módulo de deformación en el rango de cargas entre 20 y $50 \mathrm{kPa}$ en muestras equilibradas a -60hPa y secas al aire, para cada sitio, sector y profundidad. Valores promedio $( \pm \mathrm{DE})$.

Table 6. Deformation module in the load range between 20 and $50 \mathrm{kPa}$ in samples equilibrated at $-60 \mathrm{hPa}$ and air-dried, for each treatment, site and depth. Average values $( \pm \mathrm{SD})$.

\begin{tabular}{|c|c|c|c|c|}
\hline \multirow{2}{*}{$\begin{array}{c}\mathrm{N}^{\circ} \\
\text { Pasadas }\end{array}$} & \multicolumn{2}{|c|}{$-60 \mathrm{hPa}\left(\mu \mathrm{m} \mathrm{kPa}{ }^{-1}\right)$} & \multicolumn{2}{|c|}{ Seco al aire $\left(\mu \mathrm{m} \mathrm{kPa}^{-1}\right)$} \\
\hline & LC & $\mathrm{P}$ & LC & $\mathrm{P}$ \\
\hline & \multicolumn{4}{|c|}{ Superficie $(0-20 \mathrm{~cm})$} \\
\hline Previa & $40,3( \pm 22,8)$ a & $58,4( \pm 14,5)$ a & $22,5( \pm 6,2) \mathrm{a}$ & $19,0( \pm 3,9) \mathrm{a}$ \\
\hline 0 & $31,3( \pm 15,3)$ a & $99,4( \pm 15,2) b^{*}$ & $19,1( \pm 7,4) \mathrm{a}$ & $27,9( \pm 7,2) a b$ \\
\hline 1 & $42,1( \pm 8,6) a$ & $85,2( \pm 11,8) a b^{*}$ & $17,3( \pm 4,4) \mathrm{a}$ & $35,4( \pm 5,9) \quad b^{*}$ \\
\hline \multirow[t]{2}{*}{3} & $47,0( \pm 8,6) a$ & $101,0( \pm 15,4) b^{*}$ & $28,8( \pm 9,0)$ a & $28,3( \pm 7,4) a b$ \\
\hline & \multicolumn{4}{|c|}{ Profundidad $(30-50 \mathrm{~cm})$} \\
\hline Previa & $46,6( \pm 12,3)$ a & $46,9( \pm 15,4)$ a & $14,8( \pm 5,6) \mathrm{a}$ & $24,6( \pm 8,5) \mathrm{a}$ \\
\hline 0 & $44,4( \pm 8,8) a$ & $81,8( \pm 35,7)$ a & $18,3( \pm 5,4) a$ & $25,3( \pm 7,5) \mathrm{a}$ \\
\hline 1 & $40,1( \pm 7,5) a$ & $73,0( \pm 13,1) a^{*}$ & $13,1( \pm 4,5) \mathrm{a}$ & $40,8( \pm 8,2) \quad b^{*}$ \\
\hline 3 & $35,8( \pm 15,4)$ a & $64,2( \pm 6,7) a^{*}$ & $18,3( \pm 3,6)$ a & $26,2( \pm 3,7) a b^{*}$ \\
\hline
\end{tabular}

Promedios unidos por letras minúsculas distintas en sentido vertical indican diferencias estadísticamente significativas entre tratamientos $\left(\mathrm{n}^{\circ}\right.$ de pasadas) dentro de cada sitio y profundidad, según el test de Tukey (p-valor $\left.<0,05\right)$. Asterisco $(*)$ indica diferencias estadísticamente significativas en sentido horizontal entre los sitios en cada $n^{\circ}$ de pasadas y profundidad. LC: Labranza convencional; P: Pradera.

Los módulos de deformación para las muestras en condición seca al aire fueron considerablemente menores a aquellos equilibrados a $-60 \mathrm{hPa}$, lo que se explica por las tensiones internas que se generan a nivel de agregado cuando éstos se encuentran secos (Nearing, 1995). Fuentes et al. (2013b) señalan que la porosidad al interior de los agregados está asociada a un bajo diámetro y un alto grado de tortuosidad, lo que genera una mayor estabilidad producto de las tensiones internas que se generan en la condición seca.

\section{Módulo de deformación entre 300 y 500 kPa}

En el tramo de cargas altas, la consolidación es mucho menor (Cuadro 7), ya que se ha reducido gran parte del espacio poroso (Horn et al., 2007). En las muestras superficiales equilibradas a $-60 \mathrm{hPa}$ se generaron diferencias significativas entre sitios (LP v/s P) para la condición previa a la labranza y luego de la tercera pasada de tractor, lo que se repite en profundidad en la tercera pasada de tractor; en todos los casos siempre el sitio LC tiene un menor módulo de deformación, en respuesta a los mayores niveles de densidad aparente, lo que denota un estado previo de consolidación mayor (Rücknagel et al., 2007). Las maquinarias de cosecha generan una presión promedio de entre 150 y $250 \mathrm{kPa}$ sobre el suelo; por otro lado, las máquinas que más presión ejercen son las de carga con un solo eje, como algunos carros de acopio y transporte, pudiendo llegar a los $650 \mathrm{kPa}$ de presión (Febo y Pessina,
2002); así, resulta crucial el estado de humedad del suelo al momento del tránsito para evitar su degradación.

La labranza tiene un efecto significativo sobre este módulo en el sitio $\mathrm{P}$ entre $0-20 \mathrm{~cm}$, donde la soltura mecánica y la posterior estabilidad del sistema poroso permite que el suelo se siga deformando frente a cargas excesivas. Al comparar los resultados de las muestras en superficie y en profundidad, se observa que ambas tienen módulos similares, lo que indica que con esas magnitudes el suelo pierde sus características, comportándose como un sistema homogéneo.

La deformación del suelo depende de distintos factores; es así como bajo condiciones de saturación, la curva de consolidación dependerá principalmente del estrés externo y la continuidad de poros para la evacuación del agua (Nearing, 1995), mientras que bajo condiciones no saturadas, las tensiones internas generan una mayor resistencia en el suelo (Horn, 2003), efecto que se observó en los rangos de carga baja (Cuadro 6). Por otra parte, mientras más gruesa sea la textura, más alta será la densidad aparente y más baja la tensión mátrica, esperándose un menor estrés por compresión; por otra parte, la cantidad de agua drenada durante una prueba de consolidación es afectada no solo por la textura del suelo, la densidad aparente y el contenido inicial de agua, sino también por el tiempo del intervalo de carga (Krümmelbein et al., 2008).

Se ha observado que a mayor contenido de arcilla y agua, más grande será la compresibilidad, pero esta in- 
Cuadro 7. Módulo de deformación comprendido entre los 300 y $500 \mathrm{kPa}$ en muestras equilibradas a -60hPa y secas al aire, para cada tratamiento, sitio y profundidad. Valores promedios $( \pm \mathrm{DE})$.

Table 7. Deformation module in the load range between 300 and $500 \mathrm{kPa}$ in air-dried samples equilibrated at - $60 \mathrm{hPa}$, for each treatment, site and depth. Average values $( \pm \mathrm{SD})$.

\begin{tabular}{|c|c|c|c|c|}
\hline \multirow{2}{*}{$\begin{array}{c}\mathrm{N}^{\circ} \\
\text { Pasadas }\end{array}$} & \multicolumn{2}{|c|}{$-60 \mathrm{hPa}\left(\mu \mathrm{m} \mathrm{kPa}{ }^{-1}\right)$} & \multicolumn{2}{|c|}{ Seco al aire $\left(\mu \mathrm{m} \mathrm{kPa}^{-1}\right)$} \\
\hline & LC & $\mathrm{P}$ & LC & $\mathrm{P}$ \\
\hline & \multicolumn{4}{|c|}{ Superficie $(0-20 \mathrm{~cm})$} \\
\hline Previa & $5,6( \pm 0,6)$ a & $6,8( \pm 0,5) a^{*}$ & $4,4( \pm 1,1) \mathrm{a}$ & $3,2( \pm 0,7)$ a \\
\hline 0 & $5,0( \pm 1,0)$ a & $8,0( \pm 3,4)$ a & $5,1( \pm 1,1)$ a & $5,2( \pm 0,5) \quad b$ \\
\hline 1 & $4,3( \pm 0,6)$ a & $6,1( \pm 1,3)$ a & $4,4( \pm 0,7)$ a & $5,2( \pm 1,1) \quad b$ \\
\hline \multirow[t]{2}{*}{3} & $4,3( \pm 0,4)$ a & $6,2( \pm 0,8) a^{*}$ & $3,8( \pm 1,5) \mathrm{a}$ & $5,7( \pm 0,9) \quad b^{*}$ \\
\hline & \multicolumn{4}{|c|}{ Profundidad $(30-50 \mathrm{~cm})$} \\
\hline Previa & $5,4( \pm 1,2)$ a & $5,2( \pm 0,7)$ a & $4,5( \pm 1,1)$ a & $3,8( \pm 1,3)$ a \\
\hline 0 & $4,5( \pm 0,3)$ a & $5,3( \pm 0,8)$ a & $3,7( \pm 1,2) \mathrm{a}$ & $4,5( \pm 1,3)$ a \\
\hline 1 & $4,9( \pm 2,5)$ a & $6,0( \pm 1,1)$ a & $3,0( \pm 0,3)$ a & $4,7( \pm 1,8)$ a \\
\hline 3 & $4,8( \pm 0,5)$ a & $6,1( \pm 0,6) a^{*}$ & $3,8( \pm 1,4)$ a & $5,7( \pm 8,0)$ a \\
\hline
\end{tabular}

Promedios unidos por letras minúsculas distintas en sentido vertical indican diferencias estadísticamente significativas entre tratamientos $\left(\mathrm{n}^{\circ}\right.$ de pasadas) dentro de cada sitio y profundidad, según el test de Tukey ( $\mathrm{p}$ valor $\left.<0,05\right)$. Asterisco $(*)$ indica diferencias estadísticamente significativas en sentido horizontal entre los sitios en cada ${ }^{\circ}$ de pasadas y profundidad. LC: Labranza convencional; P: Pradera.

teracción puede modificarse con el grado de desarrollo estructural (Seguel y Horn, 2006b; Seguel y Orellana, 2008). Así, existe una mayor resistencia de agregados húmedos en suelos con alto contenido de $\mathrm{MO}$ que en aquellos con bajo contenido de MO, debido a su comportamiento hidrofóbico, que ralentiza la humectación y aumenta la estabilidad (Zhang et al., 2005; Fuentes et al., 2013a). También se ha observado que para el mismo suelo y contenido de agua, un aumento en la densidad aparente se asocia con un aumento en la resistencia (Horn, 2003); sin embargo, para diferentes suelos esta relación no es válida, y el diámetro de los poros (relacionado con la textura de los suelos) tendría mayor influencia en la resistencia (Fuentes et al., 2013b), lo que depende de la estructura y la fuerza de unión entre los agregados (Seguel y Orellana 2008). Esto explicaría las diferencias entre las muestras superficiales de P, donde la soltura mecánica generada por la labranza destruyó las uniones entre agregados, los que poseen una alta resistencia individual, permitiendo la persistencia de poros intra-agregados que se siguen asentando con altas presiones.

\section{Módulo de recuperación}

Respecto a la recuperación del suelo post carga (Cuadro 8), en las muestras equilibradas a $-60 \mathrm{hPa}$ no se registraron diferencias significativas entre tratamientos. Por otro lado, las muestras que corresponden al sitio P siempre alcanzaron una mayor recuperación en comparación con aquellas del sitio LC en ambas profundidades, aunque solo con diferencias estadísticamente significativas previo a la labranza y posterior a la tercera pasada entre 0 y $20 \mathrm{~cm}$. La resiliencia de un suelo depende de sus características inherentes y de su calidad estructural, es por esto que el sitio P presentó los mayores valores de recuperación, ya que posee un ligero mayor contenido de arcilla, lo que sumado al mayor contenido de MO le confiere la capacidad de recuperación elástica (Zhang et al., 2005).

Otro factor a considerar en la recuperación de un suelo es la readsorción de agua, ya que las cargas aplicadas en la prueba de consolidación, al modificar la porosidad del suelo, generan cambios en el potencial mátrico. Al respecto, Seguel y Horn (2006a) determinaron que cargas bajas no generan grandes cambios en el potencial hidráulico, pero al seguir aumentando las cargas, se produce un aumento sostenido de éste debido al colapso del sistema macroporoso (con una neoformación de meso y microporos), con la consecuente liberación de agua; finalmente, al liberar las cargas, se produce el aumento de volumen de suelo y la disminución del potencial hidráulico, con el consecuente incremento de la resistencia.

En las muestras secas al aire y en superficie del sitio LC, se generaron diferencias significativas entre la condición post-labranza y la tercera pasada, ya que después de la labranza la estructura es inestable, debido 
Cuadro 8. Módulo de recuperación comprendido entre los 500 y 0 kPa en muestras equilibradas a -60hPa y secas al aire, para cada sitio, sector y profundidad. Valores promedio $( \pm \mathrm{DE})$.

Table 8. Recovery module in the load range between 500 and $0 \mathrm{kPa}$ in air-dried samples equilibrated at $-60 \mathrm{hPa}$, for each treatment, site and depth. Average values $( \pm \mathrm{SD})$.

\begin{tabular}{|c|c|c|c|c|}
\hline \multirow{2}{*}{$\begin{array}{c}\mathrm{N}^{\circ} \\
\text { Pasadas }\end{array}$} & \multicolumn{2}{|c|}{$-60 \mathrm{hPa}\left(\mu \mathrm{m} \mathrm{kPa} \mathrm{a}^{-1}\right)$} & \multicolumn{2}{|c|}{ Seco al aire $\left(\mu \mathrm{m} \mathrm{kPa}{ }^{-1}\right)$} \\
\hline & $\mathrm{LC}$ & $P$ & $\mathrm{LC}$ & $P$ \\
\hline & \multicolumn{4}{|c|}{ Superficie $(0-20 \mathrm{~cm})$} \\
\hline Previa & $0,9( \pm 0,2) a$ & $2,0( \pm 0,2) a^{*}$ & $0,7( \pm 0,5) a b$ & $0,9( \pm 0,1) \mathrm{a}$ \\
\hline 0 & $1,2( \pm 0,6) \mathrm{a}$ & $1,9( \pm 0,2) a$ & $0,1( \pm 0,2) \mathrm{a}$ & $0,7( \pm 0,6) \mathrm{a}$ \\
\hline 1 & $1,0( \pm 0,2) \mathrm{a}$ & $1,7( \pm 1,1) \mathrm{a}$ & $0,8( \pm 0,4) a b$ & $0,9( \pm 0,7) \mathrm{a}$ \\
\hline \multirow[t]{2}{*}{3} & $1,1( \pm 0,3) \mathrm{a}$ & $2,3( \pm 0,6) a^{*}$ & $1,3( \pm 0,2) \quad b$ & $0,5( \pm 0,5) \mathrm{a}^{*}$ \\
\hline & \multicolumn{4}{|c|}{ Profundidad $(30-50 \mathrm{~cm})$} \\
\hline Previa & $0,9( \pm 0,3)$ a & $1,3( \pm 0,4) \mathrm{a}$ & $0,5( \pm 0,3) \mathrm{a}$ & $0,5( \pm 0,3)$ a \\
\hline 0 & $1,1( \pm 0,3) \mathrm{a}$ & $1,7( \pm 0,8) \mathrm{a}$ & $0,8( \pm 0,5)$ a & $0,8( \pm 0,6) \mathrm{a}$ \\
\hline 1 & $1,4( \pm 0,3) \mathrm{a}$ & $1,6( \pm 0,1) a$ & $1,2( \pm 0,4) \mathrm{a}$ & $0,7( \pm 0,8) \mathrm{a}$ \\
\hline 3 & $1,2( \pm 0,8) \mathrm{a}$ & $1,4( \pm 0,3) \mathrm{a}$ & $1,0( \pm 0,2) \mathrm{a}$ & $0,6( \pm 0,4) \mathrm{a}$ \\
\hline
\end{tabular}

Promedios unidos por letras minúsculas distintas en sentido vertical indican diferencias estadísticamente significativas entre tratamientos $\left(\mathrm{n}^{\circ}\right.$ de pasadas) dentro de cada sitio y profundidad, según el test de Tukey ( $\mathrm{p}$-valor $\left.<0,05\right)$. Asterisco $(*)$ indica diferencias estadísticamente significativas en sentido horizontal entre los sitios en cada $n^{\circ}$ de pasadas y profundidad. LC: Labranza convencional; P: Pradera.

a la pérdida de puntos de contacto entre las partículas (Horn et al., 2007), determinando que el suelo presente una muy baja recuperación al ser sometido a cargas en condiciones de laboratorio. Por otro lado, el mayor número de pasadas genera un mayor reacomodo de las partículas, aumentando los puntos de contacto, lo que previene que la aplicación de la carga de $500 \mathrm{kPa}$ genere la máxima deformación (Cuadro 5). Esta condición permite que se manifieste la plasticidad natural de las partículas; sin embargo, la capacidad de recuperación se logra a costa de la pérdida de poros funcionales (Seguel y Orellana, 2008). Lo anterior no ocurre en el sitio $\mathrm{P}$, donde la recuperación es estable a lo largo de los tratamientos gracias a su calidad estructural. Pese a esto, en la tercera pasada de tractor en condición seco al aire existieron diferencias significativas entre ambos sitios, con una mayor recuperación en el sitio LC. Lo anterior da cuenta de lo determinante que es el contenido de agua para la recuperación del sitio $\mathrm{P}$, mientras que las muestras del sitio LC parecen ser menos dependientes de este factor.

\section{Capacidad de soporte}

Los resultados de esta propiedad indican que, para la condición húmeda $(-60 \mathrm{hPa})$, tanto en superficie como en profundidad las muestras del sitio $\mathrm{P}$ bajaron significativamente su capacidad de soporte tras la labranza, efecto que se mantuvo con los posteriores trán- sitos de tractor (Figura 2). Por otro lado, el sector LC también disminuyó su capacidad de soporte entre 0-20 $\mathrm{cm}$, pero sin diferencias estadísticas significativas.

En el sitio LC, el hecho de humectar las muestras para luego equilibrarlas a $-60 \mathrm{hPa}$ genera un asentamiento debido a su menor estabilidad estructural (Seguel et al., 2006a); este asentamiento le permite resistir de mejor manera las cargas en la prueba de consolidación, ya que se genera un rápido incremento de la densidad aparente, con el consecuente aumento de la resistencia (Rücknagel et al., 2007). En el sitio P la humectación no produce este asentamiento previo, pero durante la prueba de consolidación el agua funciona como un agente lubricante que disminuye la capacidad de soporte (Tang et al., 2009).

En condiciones secas al aire no se generaron diferencias significativas entre tratamientos y sitios. Los valores de capacidad de soporte en el sitio LC seco al aire son mayores a aquellos determinados por Seguel y Horn (2006a) para un Mollisol de Chile central con historial de labranza permanente. En dicho estudio se obtuvieron valores de 23 y $45 \mathrm{kPa}$ en superficie (0-10 $\mathrm{cm})$ y profundidad $(40-60 \mathrm{~cm})$ respectivamente, resultado que denota la importancia de la frecuencia e intensidad de labranza sobre las propiedades físicas del suelo (Alleto y Coquet, 2009).

A modo general, el sitio LC presentó una mayor capacidad de soporte que el sitio $\mathrm{P}$, lo que puede estar relacionado al tamaño de poros y su estabilidad, ya 


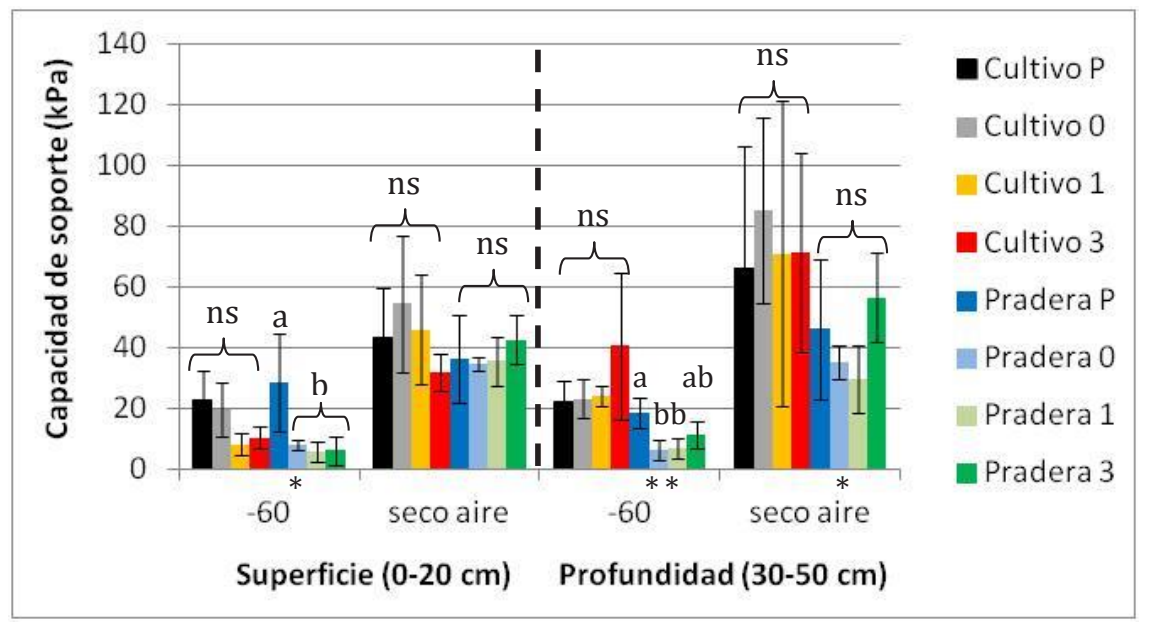

Figura 2. Capacidad de soporte en muestras equilibradas a $-60 \mathrm{hPa}$ y secas al aire, para cada tratamiento, sitio y profundidad. Valores promedios $( \pm \mathrm{DE})$. Valores unidos por letras minúsculas distintas indican diferencias estadísticamente significativas entre tratamientos $\left(\mathrm{n}^{\circ}\right.$ de pasadas) dentro de cada sitio y profundidad (test de Tukey, p-valor $\left.<0,05\right)$. Asterisco $\left({ }^{*}\right)$ indica diferencias estadísticamente significativas entre los sitios en cada $n^{\circ}$ de pasadas y profundidad.

Figure 2. Loading capacity in air-dried and equilibrated at $-60 \mathrm{hPa}$ samples, for each treatment, site and depth. Average values $( \pm$ SD). Values with different lowercase letter denote statistically significant differences between treatments ( $\mathrm{Nr}$ of passes) in each site and depth (Tukey test, p-value $<0.05)$. Asterisk $\left(^{*}\right)$ denote statistically significant differences between sites in each $\mathrm{Nr}$ of passes and depth.

que poros más grandes requieren de una menor carga para colapsar que aquellos más pequeños (Horn, 2003). En relación a lo anterior, Fuentes et al. (2013a) compararon la capacidad de soporte de agregados en dos condiciones (sin intervención y bajo labranza) en dos Mollisoles de Chile central, donde en ambos suelos la mayor capacidad de soporte la alcanzó la condición bajo labranza. En el sitio P del presente estudio, la mejor calidad física de la estructura favorece un estado friable de alta porosidad, lo que a nivel de estabilidad mecánica responde a una baja capacidad de soporte, especialmente post-labranza y en condición húmeda.

\section{CONCLUSIONES}

El mayor contenido de materia orgánica en el sector manejado con pradera determinó una mejor estructuración, la cual permitió conservar el efecto positivo de la labranza, con menores valores de densidad aparente y mayor nivel de macroporosidad en relación al sitio manejado con labranza convencional tras ser transitados.

En las pruebas de consolidación, el sitio bajo labranza convencional fue el que alcanzó en general los menores módulos de deformación y la mayor capacidad de soporte, como resultado del deterioro estructural de este manejo por efecto de la labranza y posterior tránsito, resultando en una alta densidad aparente, bajo porcentaje de macroporosidad y una alta resistencia mecánica.
El sitio de pradera presentó un predominio de macro y meso porosidad, que si bien son positivos desde el punto de vista agronómico, determinaron un suelo más sensible a cargas mecánicas externas posterior a la labranza. Finalmente, tras liberar la carga, el sitio bajo pradera tuvo una mayor recuperación elástica que el de labranza convencional, como resultado del mayor contenido de materia orgánica.

\section{REFERENCIAS}

Alleto, L., Coquet, Y., 2009. Temporal and spatial variability of soil bulk density and near-saturated hydraulic conductivity under two contrasted tillage management systems. Geoderma 152, 85-94. https://doi.org/10.1016/j.geoderma.2009.05.023

Arvidsson, J., Keller, T., 2007. Soil stress as affected by wheel load and tyre inflation pressure. Soil and Tillage Research 96(2), 284-291. https://doi.org/10.1016/j. still.2007.06.012

Barto, E., Alt, F., Oelmann, Y., Wilcke, W., Rillig, M., 2010. Contributions of biotic and abiotic factors to soil aggregation across a land use gradient. Soil Biology and Biochemistry 42(12), 2316-2324. https://doi.org/10.1016/j. soilbio.2010.09.008

Chenu, C. Plante, F., 2006. Clay-sized organo-mineral complexes in a cultivation chronosequence: revisiting the concept of the "primary organo-mineral complex". European Journal of Soil Science 57, 596-607. https://doi. org/10.1111/j.1365-2389.2006.00834.x

Centro de Información de Recursos Naturales (CIREN), 1996. 
Estudio Agrológico Región Metropolitana. Descripciones de Suelos Materiales y Símbolos. Publicación CIREN $\mathrm{N}^{\circ} 115$, Santiago, Chile.

Dörner, J., Dec, D., Zúñiga, F., Horn, R., López, I., Leiva, C., Cuevas, J., 2013. Changes in the physical quality of an Andosol under different management intensities in Southern Chile, in: Krümmelbein, J., Horn, R., Pagliai, M. (Eds.), Soil degradation. Advances in Geoecology, Vol. 42, Reiskirchen, Germany, pp. 262-281.

Ellies, A., Dörner, J., 1999. Distribución de tensiones en un suelo Palehumult ejercida con el tráfico de la maquinaria agrícola. Agro Sur 27(2), 1-9.

Ernst, O., Siri-Prieto, G., 2009. Impact of perennial pasture and tillage systems on carbon input and soil quality indicators. Soil and Tillage Research 105(2), 260-268. https://doi.org/10.1016/j.still.2009.08.001

Febo, P., Pessina, D., 2002. Soil compaction: The ground pressure exerted by agricultural machines, in: Pagliai, M., Jones, R. (Eds.), Sustainable land management - environmental protection: A soil physical approach. Advances in Geoecology. Vol. 35. Reiskirchen, Germany, pp. 339-350.

Franzluebbers, A., 2002. Soil organic matter stratification ratio as an indicator of soil quality. Soil and Tillage Research 66(2), 95-106. https://doi.org/10.1016/S01671987(02)00018-1

Fuentes, I., Seguel, O., Casanova, M., 2013a. Elasto-plastic behaviour of soil aggregates and the soil matrix as a function of physical properties in three soils of central Chile, in: Krümmelbein, J., Horn, R., Pagliai, M. (Eds.), Soil degradation. Advances in Geoecology, Vol. 42, Reiskirchen, Germany, pp. 72-88.

Fuentes, I., Seguel, O., Casanova, M., 2013b. Shear strength of aggregates compared with bulk soil of two haploxerolls from Chile. Journal of Soil Science and Plant Nutrition 13(4), 819-831. http://dx.doi.org/10.4067/S071895162013005000065

Håkansson, I., 2005. Machinery-induced soil compaction of arable soil. Incidence, consequences, counter-measures. Reports from the Division of Soil Management Nr 109. Uppsala, Swedish.

Głąb, T., Kulig, B., 2008. Effect of mulch and tillage system on soil porosity under wheat (Triticum aestivum). Soil and Tillage Research 99(2), 169-178. https://doi. org/10.1016/j.still.2008.02.004

Hartge, K., Horn, R., 2009. Die physikalische Untersuchung von Boden. Praxis Messmethoden Auswertung. Überarbeitete Auflage. Schweizerbart Vorlage, Stuttgart.

Horn, R., 2003. Stress-strain effects in structured unsaturated soils on coupled mechanical and hydraulic processes. Geoderma 116(1), 77-88. https://doi.org/10.1016/ S0016-7061(03)00095-8

Horn. R., Hartge, K., Bachmann, J., Kirkham, M., 2007. Mechanical stresses in soils assessed from bulk-density and penetration-resistance data sets. Soil Science Society of America Journal 71(5), 1445-1459. https:// doi:10.2136/sssaj2006.0044

Horn, R., Fleige, H., 2009. Risk assessment of subsoil compaction for arable soils in Northwest Germany at farm scale. Soil and Tillage Research 102(2), 201-208. https://doi. org/10.1016/j.still.2008.07.015

Jorajuría, D., 2005. Compactación del suelo agrícola inducida por tráfico vehicular. Una revisión, en: Jorajuría, D. (Ed.) Reología del suelo agrícola bajo tráfico. La Plata, Argentina, pp. 39-55.

Krümmelbein, J., Peth, S., Horn, R., 2008. Determination of pre-compression stress of a variously grazed steppe soil under static and cyclic loading. Soil and Tillage Research 99, 139-148. https://doi.org/10.1016/j. still.2008.01.008

Mordhorst, A., Zimmermann, I., Peth, S., Horn, R., 2012. Effect of hydraulic and mechanical stresses on cyclic deformation processes of a structured and homogenized silty Luvic Chernozem. Soil and Tillage Research 125, 3-13. https://doi.org/10.1016/j.still.2012.06.008

Mulumba, L., Lal, R., 2008. Mulching effects on selected soil physical properties. Soil and Tillage Research 98, 106111. https://doi.org/10.1016/j.still.2007.10.011

Nearing, M., 1995. Compressive strength for an aggregated and partially saturated soil. Soil Science Society of America Journal 59(1), 35-38. https://doi:10.2136/sssaj19 95.03615995005900010005x

Osunbitan, J., Oyedele, D., Adekalu, K., 2005. Tillage effects on bulk density, hydraulic conductivity and strength of a loamy sand soil in southwestern Nigeria. Soil and Tillage Research 82(1), 57-64. https://doi.org/10.1016/j. still.2004.05.007

Payahuala, M., Dörner, J., 2009. Determinación de la capacidad de soporte de un Andisol (Duric Hapludand) por medio de dos métodos gráficos. Agro Sur 37(3), 220231. https://doi:10.4206/agrosur.2009.v37n3-06

Reynolds, W., Drury, C., Tan, C., Fox, C., Yang, X., 2009. Use of indicators and pore volume-function characteristics to quantify soil physical quality. Geoderma 152, 252-263. https://doi.org/10.1016/j.geoderma.2009.06.009

Rücknagel, J., Hofmann, B., Paul, R., Christen, O., Hülsbergen, K., 2007. Estimating precompression stress of structured soils on the basis of aggregate density and dry bulk density. Soil and Tillage Research 92(1-2), 213-220. https://doi.org/10.1016/j.still.2006.03.004

Sadzawka, A., Carrasco, M., Grez, R., Mora, M., Flores, H., Neaman, A., 2006. Métodos de análisis recomendados para los suelos de Chile. Instituto de Investigaciones Agropecuarias. Serie Actas INIA N ${ }^{\circ} 34$, Santiago, Chile.

Salvo, L., Hernández, J., Ernst, O., 2010. Distribution of soil organic carbon in different size fractions, under pasture and crop rotations with conventional tillage and notill systems. Soil and Tillage Research 109(2), 116-122. https://doi.org/10.1016/j.still.2010.05.008

Sandoval, M., Dörner, J., Seguel, O., Cuevas, J., Rivera, D., 2012. Métodos de análisis físicos de suelos. Departamento de Suelos y Recursos Naturales Universidad de Concepción. Publicación $\mathrm{N}^{\circ}$ 5, Chillán, Chile.

Schäffer, B., Attinger, W., Schulin, R., 2007. Compaction of restored soil by heavy agricultural machinery. Soil physical and mechanical aspects. Soil and Tillage Research 93(1), 28-43. https://doi.org/10.1016/j.still.2006.03.007

Schäffer, B., Boivin, P., Schulin, R., 2010. Compressibility of repacked soil as affected by wetting and drying between uniaxial compression tests. Soil Science Society of America Journal 74(5), 1483-1492. https://doi:10.2136/ sssaj2009.0381

Schoeneberger, P., Wysocki, D., Benham, E., 2012. Field book 
for describing and sampling soils. Natural Resources Conservation Service. Lincoln, Nebraska.

Seguel, O., García de Cortázar, V., Casanova, M., 2003. Variación en el tiempo de las propiedades físicas de un suelo con adición de enmiendas orgánicas. Agricultura Técnica (Chile) 63, 287-297.

Seguel, O., Horn, R., 2006a. Structure properties and pore dynamics in aggregate beds due to wetting-drying cycles. Journal of Plant Nutrition and Soil Science 169(2), 221232. https://doi.org/10.1002/jpln.200521854

Seguel, O., Horn, R., 2006b. Strength regain in soil aggregate beds by swelling and shrinkage. International Agrophysics 20, 161-172.

Seguel, O., Orellana, I., 2008. Relación entre las propiedades mecánicas de suelos y los procesos de génesis e intensidad de uso. Agro Sur 36(2), 82-92. https://doi:10.4206/ agrosur.2008.v36n2-05

Seguel, O., Baginsky, C., Contreras, A., Covarrubias, J., González, C., Sandoval, M., 2011. Changes in physical properties of a Typic Haplocambid by anual crop culture. Journal of Soil Science and Plant Nutrition 11(1), 1-15. https://doi.org/10.4067/S0718-95162011000100001

Six, J., Bossuyt, H., Degryze, S., Denef, K., 2004. A history of research on the link between (micro) aggregates, soil biota, and soil organic matter dynamics. Soil and Tilla- ge Research 79(1), 7-31. https://doi.org/10.1016/j. still.2004.03.008

Tang, A., Cui, Y., Eslami, J., Défossez, P., 2009. Analysing the form of the confined uniaxial compression curve of various soils. Geoderma 148, 282-290. https://doi. org/10.1016/j.geoderma.2008.10.012

Taylor, H., Brar, G., 1991. Effect of soil compaction on root development. Soil and Tillage Research 19(2-3), 111-119. https://doi.org/10.1016/0167-1987(91)90080-H

Uribe, J., Cabrera, R., de la Fuente, A., Paneque, M., 2012. Atlas bioclimático de Chile. Santiago, Chile.

Usowicz, B., Lipiec, J., 2009. Spatial distribution of soil penetration resistance as affected by soil compaction: The fractal approach. Ecological Complexity 6(3), 263-271. https://doi.org/10.1016/j.ecocom.2009.05.005

Vaz, C., Manieria, J., de Maria, I.C., Tuller, M., 2011. Modeling and correction of soil penetration resistance for varying soil water content. Geoderma 166, 92-101. https://doi. org/10.1016/j.geoderma.2011.07.016

Warrick, A., 2002. Soil physics companion. CRC Press. New York, USA.

Zhang, B., Horn, R., Hallett, P., 2005. Mechanical resilience of degraded soil amended with organic matter. Soil Science Society of America Journal 69(3), 864-871. https://doi. org/10.2136/sssaj2003.0256 
